\title{
Valoración del uso de Portafolio Electrónico en la Formación Inicial Docente
}

\author{
Brenda Angélica Lara Subiabre ${ }^{1}$ \\ María Raquel Pereira Berríos ${ }^{2}$ \\ Universidad de Los Lagos, Chile \\ Recepción: 14-09-2016 / Aceptación: 28-09-2016
}

Resumen

En el contexto de la Sociedad del Conocimiento se hace imprescindible que los futuros profesores se apropien de las tecnologías de la información y la comunicación (TIC), para responder a los requerimientos de los aprendices del nuevo milenio. El objetivo de la investigación fue establecer la valoración que le dan los estudiantes de la carrera de Educación Física al uso del portafolio electrónico. La metodología fue cualitativa, de tipo descriptivo con un diseño fenomenológico. En primera instancia se recogió información a través de un grupo focal y entrevistas, con los que posteriormente se elabora una encuesta semi-estructurada. Los participantes finales fueron un grupo homogéneo y correspondieron a 56 estudiantes de los últimos dos años de formación.

Los resultados dan cuenta de factores que ellos valoran al usar el portafolio, tales como: intercambio de ideas, resolver dudas, revisar trabajos producidos por ellos mismos y por compañeros. Además manifiestan que les desagradan, aspectos del diseño del software, capacidad del sistema y la visualización de su información en buscadores de Internet. Se concluye que los estudiantes reconocen una alta valoración del uso del portafolio, lo que puede favorecer su disposición a trabajar con tecnología una vez que sean profesores.

Palabras clave: portafolio electrónico, formación inicial docente, evaluación de competencias, Tecnologías de la Información y la Comunicación.

\begin{abstract}
In the context of Knowledge Society, it is essential that future teachers make theirs the Information and Communication Technology (ICT) in order to respond to the learners' requirements of the new millennium. The objective of this research was to establish the valuation given by students of the major Physical Education in means of the use of electronic portfolio. The methodology was qualitative, in the descriptive type with a phenomenological design. First of all, information was gathered through a focus group and interviews, from which, later, a semi-structured survey was created. The final participants were a homogenous group, and it corresponded to 56 students who are in their last two years of training.

The results show the matters they valuate when using the portfolio, such as: exchange of ideas, solving doubts, revision of works made by themselves and by classmates. Moreover, they say they dislike the aspects of the software design, system capacity, and the visualization of its information in internet search engines. It is concluded that the students recognize a high valuation of the use of the portfolio, which can favor their willingness toward working with technology once they become teachers.
\end{abstract}

Key Words: electronic portfolio, initial teacher training, competence assessment, Information and Comunication Technologies.

1. Académica; email: blara@ulagos.cl

2. Profesora de Estado en Educación Física; email: mpereira@ulagos.cl 


\section{Introducción}

Desde su creación en el año 2005, la carrera de Pedagogía Educación Media, mención Educación Física, ha buscado formas de enseñar y evaluar competencias definidas en su perfil de egreso, tarea que no ha estado exenta de dificultades, debido a que fue una de las primeras tres carreras que se crean en la Universidad de Los Lagos (ubicada en la ciudad de Puerto Montt, Chile), bajo el modelo curricular "basado en competencias"; y tanto la institución como el equipo de docentes no tenían experiencia en la implementación de este tipo de currículum.

Si bien en el año 2006 se publicó un decreto universitario ( ${ }^{\circ} 5365$, del 23 de septiembre) que orienta la evaluación de competencias, ello no se traduce inmediatamente en una aplicación efectiva, ya que requiere de un cambio en la forma de plantear el proceso de enseñanza y aprendizaje. En esta búsqueda por responder al desarrollo de competencias y su adecuada evaluación (como una instancia de aprendizaje), el Consejo de carrera incorporó desde el año 2008 el uso de un portafolio electrónico, el que actualmente se utiliza en diferentes módulos de la malla curricular.

A seis años de su aplicación, surge la preocupación por el desconocimiento que se tiene sobre la valoración que hacen los estudiantes del uso del portafolio electrónico. Si bien, se pueden reconocer algunas opiniones surgidas en contextos variados, ellas no son significativas para identificar qué aspectos / factores tienen mayor o menor valoración desde la perspectiva de los estudiantes. La posibilidad de mejorar el trabajo desarrollado con el portafolio se sustenta en la evaluación que se hace sobre su uso y como una estrategia para avanzar en materia de apropiación de TIC en la formación inicial docente, ya que el portafolio no es utilizado solo con una finalidad técnica, sino que además para promover procesos necesarios en la praxis pedagógica, tales como: reflexión, colaboración, organización de evidencias, entre otras.

Según el informe Mckinsey (Barber y Mourshed, 2008), uno de los factores relevantes para mejorar la educación, es potenciar la formación inicial docente y desarrollar una cultura de colaboración en el quehacer pedagógico. Por su parte la UNESCO (1998), en la declaración Mundial sobre "La Educación Superior en el siglo XXI", establecía que es indispensable mejorar procesos de planificación, de didáctica y apropiación de TIC, en la formación profesional, lo que es ratificado en la Segunda conferencia mundial (UNESCO, 2009), oportunidad en la que hacen un llamado para la acción a los Estados miembros a: "apoyar una mayor integración de las TIC y fomentar el aprendizaje abierto y a distancia, con miras a satisfacer el aumento de la demanda de educación superior" (pág. 8).

En la misma línea, la UNESCO (2013) en el Foro de Alto Nivel sobre Políticas relativas a las TIC y la Educación para Todos, planteó entre sus conclusiones, varios puntos que están en estrecha relación con este trabajo, a saber: velar por el acceso equitativo al aprendizaje 
de las TIC; comprender que la aplicación de las TIC transformará completamente el sector educativo; tener en cuenta la importancia fundamental de los docentes, en tanto que agentes esenciales de la transformación de la enseñanza; y crear comunidades de expertos, basadas especialmente en recursos educativos de libre acceso y en el uso del sistema de licencias denominado Creative Commons.

En un estudio realizado por la CEPAL (Brun, 2011), se plantea que en general en Latinoamérica hay un bajo nivel de integración de las TIC en la Formación Inicial Docente, además exponen que se evidencio que existe poca investigación en la temática. Sumado a lo anterior, la situación es más preocupante cuando los estudiantes para futuros docentes pasan por las universidades y no se logran hacer cambios profundos en su concepciones sobre los procesos de enseñanza - aprendizaje (Pozo, 2006). De igual forma, Larrondo y otros, citado en Brun (2011), plantea que "los estudiantes de pedagogía ingresan a la universidad con ciertas carencias, reflejadas en sus puntajes de selección, y egresan, después de varios años de estudio, con las mismas limitaciones". Por otra parte, Hepp (2015) expresa que "las facultades de educación de la región no están preparando a los futuros profesores para un uso curricular de TIC” (pág. 201).

Brun (2011) y Hepp (2015) coinciden en que un elemento clave para la apropiación de las TIC en los futuros docentes, es que sean integradas curricularmente, en especial a las asignaturas de su área disciplinaria. En la misma línea, la Red de Portales Educativos (RELPE, 2013) reconoce que las barreras/desafíos que se plantean a las instituciones de Educación Superior que forman a los futuros docentes, tienen relación con intervenir la cultura digital de los estudiantes, la que debería ser abordada desde tres instancias: actitud; acceso a las TIC e integración de TIC a la malla curricular.

En el caso particular de Chile, en las últimas décadas se han desarrollado e impulsado iniciativas de política pública, con la finalidad de reforzar la formación de los estudiantes de carreras de pedagogía; dentro de ello, las últimas iniciativas tienen relación con la publicación de los estándares orientadores para egresados de carreras de pedagogía en educación media (Mineduc, 2012), y la implementación de convenios de desempeños (Mineduc, 2012).

Considerando los antecedentes, se hace necesario integrar las Tecnologías de la Información y la Comunicación a la formación inicial docente, incorporando el uso de un portafolio electrónico desde una perspectiva de desarrollo profesional.

La investigación buscaba responder a la siguiente interrogante: ¿Qué valoran los estudiantes en la utilización del portafolio electrónico?, y el objetivo fue establecer la valoración que le dan al uso del portafolio electrónico los estudiantes de la carrera de Educación Física. 


\section{Modelo de apropiación de TIC (TPACK)}

El modelo "Conocimiento pedagógico tecnológico del contenido" en su sigla en inglés (TPACK), es una propuesta de Mishra y Koehler(2009) con base en los planteamientos de Lee Shulman, sobre la integración de conocimientos pedagógicos y disciplinares que deberían tener los docentes (Pedagogical Content Knowledge o PCK). Ello quiere decir cómo se debe saber enseñar un determinado tipo de contenido, así los docentes no sólo deben dominar su materia, sino que también manejar cómo enseñarla; y debido a la incorporación de las tecnologías de la información y la comunicación en educación Mishra y Koehler proponen agregar este nuevo elemento.

\section{Modelo de conocimiento pedagógico tecnológico del contenido}

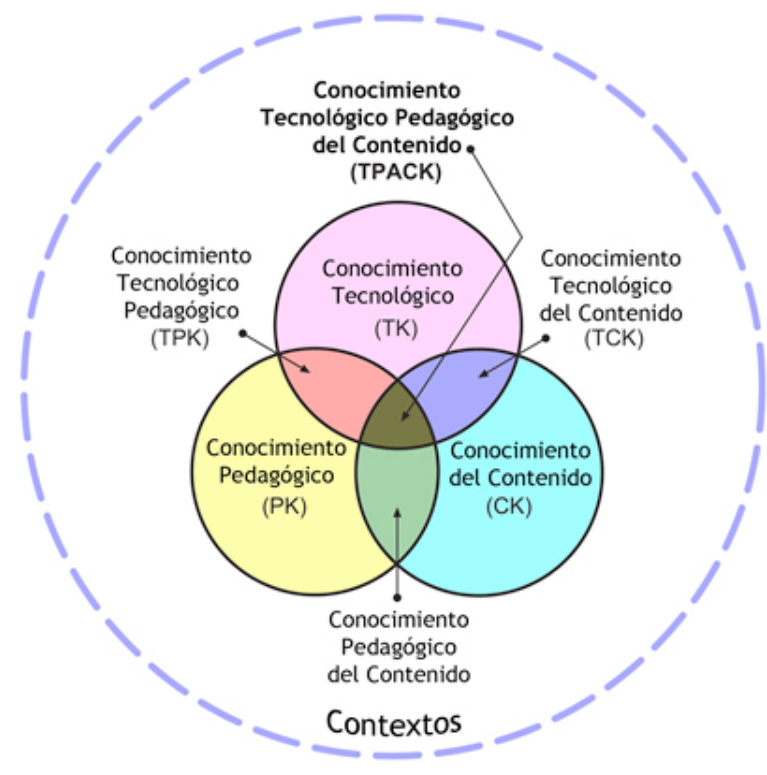

Figura 1. Modelo TPACK.

Fuente: www.tpack.org
Este modelo propone que los docentes pongan en juego sus conocimientos pedagógicos, tecnológicos y disciplinares para diseñar, implementar y evaluar situaciones de aprendizaje. Lo anterior debe considerar además las variables del contexto.

En el caso de la formación de los estudiantes de pedagogía, se ha buscado a través del portafolio la integración de los cuatro elementos, ya que como parte de su formación pedagógica se requiere que tengan vivencias en la utilización de tecnología para apoyar aprendizajes del área de Educación Física.

\section{Portafolio Electrónico}

El portafolio en formato electrónico o e-portafolio según Barberá (2008) es un sistema digital que permite a los usuarios documentar competencias o productos que han seleccionado como relevantes, de manera que muestren su progreso a través del tiempo. Los registros que forman las evidencias pueden ser de diferentes formatos (textual, audio, video, entre otros) y se presentan organizadas y acompañadas de reflexiones. El propio usuario lo controla, permaneciendo abierto para revisiones y actualizaciones, además de tener la posibilidad de retroalimentación de otros usuarios con los que se comparte. En general existe acuerdo en la literatura que los dos elementos claves de todo portafolio son: la selección y la reflexión (Barberá, 2008; Barret, 2009; Agra, Gewerc y Montero, 2003; Oner, y Adadan, 2011, 2016).

Según Barberá (2008) se pueden reconocer beneficios tanto para el usuario como para las 
organizaciones que los implementan. Entre los beneficios que se reconocen para el sujeto (usuario) están los siguientes: presentar una variedad de muestras enlazadas ágilmente y con rápido acceso; se aumenta la competencia digital del que lo utiliza; se aumenta la capacidad comunicativa y se potencia la superación; se puede transportar y es de fácil almacenamiento; entre otros. En el caso de la organización: el producto puede ser usado para mostrar las capacidades logradas por los usuarios y se almacena gran cantidad de información de fácil recuperación; entre otros.

Según Barret (2009), los portafolios se pueden clasificar de acuerdo al objetivo que persigan: a) aprendizaje o reflexión, y b) demostración o rendición de cuentas.

\section{Equilibrio entre modelos de portafolio}

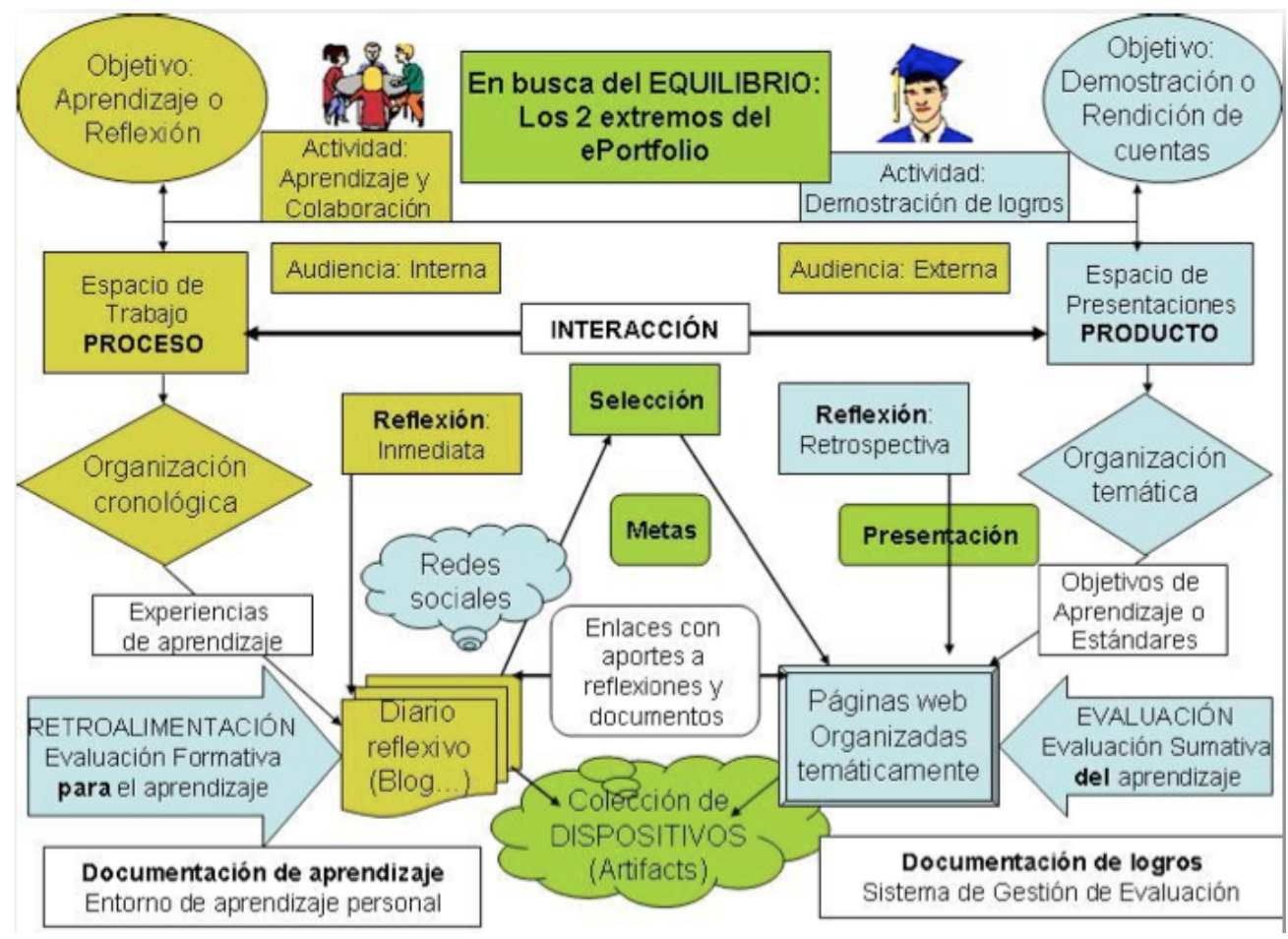

Figura 2. Equilibrios entre modelos de portafolios.

Fuente: Barrett, H. C. (2009). Recuperado de: http://electronicportafolios.org/balance 
Como se observa en la figura 2, la dinámica que se da en los portafolios es la expresión de una u otra tendencia (aprendizaje o reflexión / demostración o rendición de cuentas), por lo cual se plantea buscar un equilibrio entre ambos, por los beneficios que cada uno de ellos puede reportar.

Para efectos de esta investigación, es relevante dar a conocer el esquema que deberían tener los portafolios de profesores (Barberá, 2008), considerando que la muestra estuvo conformada por sujetos que estudian para ser docentes. Los apartados a considerar deberían ser: filosofía personal sobre la educación; planificación de las clases; materiales y recursos; metodologías y evaluación; reflexiones; y por último evidencias de desarrollo profesional.

\section{Los e-portafolios en la web 2.0}

El valor de la utilización de la web 2.0 para el desarrollo de portafolios electrónicos, principalmente obedece a fortalecer instancias de comunicación y colaboración tanto entre los docentes y estudiantes, como entre ellos. Además, este tipo de aplicaciones pueden integrar diferentes formatos (textual, video, audio, otros). Dadas las condiciones, los autores de un portafolio pueden compartir sus productos junto con comentar, argumentar y reflexionar sobre su proceso. Barret (2012) plantea un modelo para desarrollar e-portafolios utilizando herramientas de la web 2.0, que consiste en lo siguiente:

a. Primer paso: es el propósito, el que consiste en decidir la finalidad del portafolio. b. Segundo paso: colección / clasificación. ¿Qué objetos va a incluir? ¿Cómo los va a clasificar?

c. Tercer paso: reflexión. Justifican porqué esas evidencias representan el logro de resultados de aprendizaje.

d. Cuarto paso: conexión / interacción / diálogo / comentarios. Proporciona oportunidad para la interacción y la retroalimentación sobre el trabajo publicado en el portafolio.

e. Quinto paso: reflexión / selección / evaluación. El final de un proceso el estudiante elabora una reflexión que mira hacia atrás y proporciona un meta-análisis de la experiencia.

f. Sexto paso: presentación / Editorial. El autor del portafolio decide qué partes del portafolio se harán públicos.

Existe una gran variedad de softwares que son utilizados para implementar portafolios electrónicos. En este caso se ha optado por Elgg, ya que fue construido con la finalidad de ser un "software social", flexible y permite la instalación de plugins que enriquecen las herramientas de base que trae consigo. Además es una plataforma de software libre, diseñada para que los usuarios compartan recursos a través de Internet y promover la creación de redes sociales.

En el software cada usuario cuenta con sus propias herramientas para trabajar de manera personal, registrar las evidencias y escribir comentarios y/o reflexiones de su proceso, 
entre las que se cuentan las opciones de crear carpetas y subir documentos, publicar imágenes, vídeos, enlaces a web, blog, tablón de anuncios, editar el perfil, entre otras.

Otro de los aspectos importantes de destacar del software Elgg, es la posibilidad de trabajar con el modelo de redes sociales. Para ello se crea una comunidad virtual que comparte variadas herramientas, tales como: carpetas y documentos de la comunidad, blog comunitario, publicación de vídeos e imágenes y foro, entre las principales. A través de las comunidades que se forman en un portafolio es posible potenciar el diálogo con otros; este diálogo le permite al estudiante reflexionar (Oner y Adadan, 2016), además de que estando solo no puede ser capaz de verse a sí mismo, sin cierto autoengaño, limitando así el alcance de su reflexión potencial. Zeichner (1995) considera el aporte significativo de la existencia de instancias de grupo que faciliten la reflexión, ya que de manera individual se limitan en gran medida sus posibilidades de progreso. Según Solomon (en Zeichner, 1995), "El desafío crítico y el apoyo que se consigue mediante la interacción social son importantes para ayudarnos a clarificar lo que pensamos y para armarnos del valor necesario para actuar de acuerdo con lo que creemos" (pág. 391).

\section{Evaluación de Competencias}

La evaluación por competencias se genera a través de evidencias y valoraciones sobre la medida y naturaleza del progreso del estudiante, según unos resultados de aprendizaje esperados (Valverde, Revuelta y Fernández, 2012).
A continuación se presentan los criterios de calidad que deberían utilizarse a la hora de evaluar competencias, según los autores recién citados, a saber:

a. Autenticidad: los estudiantes deben percibir la tarea como relevante y significativa y considerar la competencia valiosa y transferible. El contexto físico y el tiempo de ejecución deben parecerse al requerido en el real. El contexto social debe reproducir condiciones similares, en especial lo que se espera en su forma y actitud en el desempeño. Expresar un resultado, ya sea en producto o proceso, lo que requerirá responder a varias tareas e indicadores. Los criterios de evaluación deben establecer niveles y grados de consecución, ser explícitos y transparentes para servir de guías al aprendizaje.

b. Complejidad Cognitiva: la tarea debe reflejar y el grado de habilidad cognitiva exigida. Tener en cuenta conocimientos previos y estrategias implicadas. Solicitar explicitar las razones o fundamentos de sus respuestas o de la acción escogida.

c. Imparcialidad: los estudiantes deben tener la oportunidad de demostrar sus competencias, adecuadas a su nivel, excluyendo elementos irrelevantes.

d. Significatividad: permitir a los estudiantes implicarse en problemas significativos, en los que valore su utilidad y considere pertinente para su futuro y la sociedad. 
e. Interpretación directa: el evaluador debe ser capaz de interpretar con claridad los resultados. Juzgar libre de subjetividad.

f. Transparencia: debe ser clara y comprensible para docentes y estudiantes. Además deben conocer los criterios de calificación.

g. Consecuencias educativas: la evaluación es una parte del proceso; debe ser utilizada como orientación y guía del aprendizaje.

h. Reproducibilidad de las decisiones: se puede evaluar la misma competencia con diferentes tareas, puede obtenerse combinando diferentes fuentes de información.

i. Homogeneidad: bajo condiciones de igualdad para todos los estudiantes, se puede utilizar involucrando una gran muestra de contenidos y situaciones.

j. Coste y eficacia: los esfuerzos, tiempos y recursos requeridos para la implementación del producto o proceso a evaluar debe ser justificados según los beneficios obtenidos.

En la medida que los procesos de enseñanza - aprendizaje y evaluación consideren los criterios mencionados, existirán más posibilidades de desarrollar competencias en los estudiantes y la evaluación no sólo servirá para calificar, sino también como una instancia de aprendizaje. Por otra parte, existen varios estudios donde desarrollan y evalúan competencias a través de un portafolio (Armengol, et al, 2009; Pérez, 2014; Cotta, Minardi, da Costa y de Mendonça, 2015).

\section{Metodología}

La investigación se desarrolló con un enfoque cualitativo y un diseño fenomenológico, porque se buscó estudiar el fenómeno en el contexto mismo donde se produjo y desde las perspectivas de los actores. En coherencia con lo anterior, las unidades de análisis son las opiniones que los estudiantes de la carrera de Educación Física sobre el uso que hacen del portafolio electrónico.

Los participantes fueron un grupo homogéneo y variaron en el transcurso de la investigación. Finalmente para la aplicación de la encuesta se conformó un grupo de 56 estudiantes que cumplieron con características de inclusión, tales como:

- Ser de los dos últimos años de formación de la Carrera de Educación Física,

- Haber sido parte de una o más comunidades de portafolios electrónicos o de portafolios personales creados en el transcurso de su carrera,

- Estudiantes de ambos géneros, y

- Tener un mínimo de tres años de utilizar el portafolio.

Para la recolección de la información se inició el proceso con un grupo focal y entrevista, lo que permitió configurar elementos comunes en la valoración del uso del portafolio, hallazgos que fueron la base para elaborar una encuesta semi-estructurada. Dicho instrumento, que fue sujeto a la revisión de pares 
con experiencia en la utilización del portafolio, los que entregaron sus aportes a través de una hoja de opinión, tanto en relación a la estructura, como al contenido de las preguntas. Posterior se entregó nuevamente, a juicios de expertos, para conocer si la elaboración de las preguntas contaba con el grado de credibilidad y consistencia a lo propuesto por objetivo del instrumento.

La encuesta contó con seis preguntas, con respuestas cerradas, dicotómicas y abiertas, sus preguntas se referían específicamente a los tópicos en relación al uso, qué aprenden, qué les agrada o desagrada, qué mejorarían y una opinión general acerca del portafolio electrónico. Estas categorías surgieron de las técnicas aplicadas anteriormente. Para el tratamiento de los resultados se utilizó análisis de contenido.

\section{Resultados}

Al plantear a la muestra la opción de decidir si se suprime o mantiene la utilización del portafolio, de un total de 56 respuestas el $83 \%$ prefiere continuar usándolo y una minoría (17\%) planteó que preferiría no hacerlo. Ante la consulta de ¿por qué?, la opción "sí", fundamenta en su mayoría que es porque lo usan o les sirve para: Comunicarse y compartir (con sus compañeros y profesores), manejo de información (subir-guardar-acceder a información), facilidades de uso (facilidades para entrega y acceso de trabajos y ahorro de tiempo), desarrollo de competencias profesionales (responsabilidad, manejo de tic). Entre los argumentos utilizados para que no se utilice el portafolio se reconocen las siguientes respuestas: características de la aplicación del software; ser una aplicación tipo red social; prefieren la presencialidad, que sirve para subir información y que no debería fijarse plazos.

En relación a la consulta, sí perciben que aprenden usando el portafolio, el 89\% responde positivamente, mientras un $11 \%$ lo hace de manera negativa. En el caso de los que consideran que aprenden, sus argumentos son que los ayuda la facilidad de acceso al material publicado (salir de dudas y/o ideas para complementar trabajos, además de poder acceder a materiales producidos en generaciones anteriores); en cuanto adquirir habilidades en el uso de las TIC, al hacer intercambio de ideas (hacer y recibir comentarios en los espacios dispuestos para ello).

Al solicitarles que expongan los aspectos que más les agradaba de trabajar con el portafolio electrónico, plantean que son: las posibilidades de interactuar y compartir con otros; posteriormente, la capacidad y usabilidad del software, seguido de tener un registro y respaldo de sus trabajos, estar informados y por último el acceso a material de años anteriores.

Por otra parte, igual se buscó conocer qué es lo que les desagrada del portafolio, ante lo cual manifestaron que eran los siguientes aspectos: en primer lugar, el diseño y aplicaciones del software; en segunda opción hacen referencia a aspectos de la enseñanza, tales como que sea evaluado y que no todos los profesores lo usan. Posteriormente, se presentan dos opciones que se repiten en tercer lugar donde 
está el resguardo del sistema para mantenerse activo (que no se caiga el sistema); y la capacidad del sistema para soportar la subida de un archivo. En los últimos lugares se encuentran los sentimientos de inseguridad en la publicación de archivos (no quieren que otros tengan acceso a ver lo que publicaron), y dificultad para acceder a la dirección web.

Sobre que mejorarían, predomina el diseño y aplicaciones del software (atractivo, forma de subir archivos, reducir ventanas, otros), seguido de aspectos de la enseñanza (disminuir la exigencia, libertad en la publicación de trabajos y capacitación a docentes). Por último, consideran que se debería tener mayor resguardo a la privacidad y restricciones de acceso a lo que se publican.

Al exponer una opinión general sobre el portafolio, en primera instancia plantean que es útil y facilita el proceso de aprendizaje con respuestas tales como: permite mejorar los procesos de enseñanza - aprendizaje, facilita el trabajo, simplifica las tareas, adecuado al ambiente universitario, entre otros. En segunda opción: tener acceso y posibilidades de compartir materiales que son utilizados como guía y referencia, con expresiones tales como: es un buen espacio para compartir lo que se hace en el centro de práctica, es una fuente de almacenamiento de información, se puede buscar y compartir material, hay mucho material para facilitar el trabajo y es un medio para registrar y respaldar evidencias, entre otras.

\section{Discusión}

Los resultados obtenidos dan cuenta de situaciones que ellos perciben que les facilitan el aprendizaje tales como: intercambio ideas, resolver dudas, revisar trabajos producidos por ellos mismos y por compañeros. Además, manifiestan que les genera mayor agrado tener acceso a materiales, posibilidades de interacción con otros, registrar y respaldar sus evidencias a lo largo del tiempo. Y que los factores que valoran al usar el portafolio electrónico son: que se genere interacción y colaboración; acceder a evidencias de otros compañeros; subir, organizar y respaldar evidencias; y por último, el diseño y aplicaciones del software. Coincidentemente existen similitudes entre los aspectos que valoran, lo que les agrada y aquello que consideran que los ayuda a aprender. Los hallazgos coinciden con los resultados encontrados en otros estudios con una muestra similar (Kabilan y Khan, 2012; Perea y Tarrés, 2012; Romero, 2008; Aksit, 2016; Oner y Adadan, 2011; ChiCheng, Kuo-Hung, Chaoyun y To-Yu, 2013).

Por otra parte, indican que les desagradan aspectos del diseño del software, capacidad del sistema y la visualización de su información en buscadores de Internet. Sumado a lo anterior, perciben que lo que afectaría el uso del portafolio es el diseño y aplicaciones del software. Todas ellas hacen referencia a aspectos de naturaleza tecnológica. 
Cuando los estudiantes reconocen que están aprendiendo sobre contenidos de Educación Física y tomando decisiones pedagógicas en las asignaturas que imparten, a la vez que hacen uso de las TIC, se deja en evidencia la implementación del modelo TPACK (Koehler y Moshra, 2009).

En los aspectos valorados se reconoce estrecha relación con los beneficios del uso de un portafolio electrónico planteados por Barbera (2008); en especial al expresar que puede publicar y acceder a una variedad de muestras de materiales y trabajos, y con fácil acceso, que mejoran competencia TIC; se aumentan los espacios de comunicación; es de fácil almacenamiento, entre otros. En el caso de la carrera de Pedagogía en Educación Física ha logrado recolectar una gran variedad de producto de aprendizaje con los que pueden mostrar las capacidades logradas por los estudiantes, y se almacena gran cantidad de información de fácil recuperación. Además, el que perciban la utilidad de las TIC nos puede predecir mayores probabilidades de uso al ser profesionales (Badia, Chumpitaz, Vargas y Suárez, 2016).

De acuerdo con la búsqueda de equilibrio planteado por Barret (2009) entre los polos: "demostración y rendición de cuentas" y “aprendizaje o reflexión", los estudiantes expresan valorar ambas situaciones, tanto la posibilidad de interactuar y compartir con sus compañeros y profesores, como el poder subir, organizar y acceder a evidencias que son no solo producto final, sino que también dan cuenta de un proceso.
Según el criterio de autenticidad (Valverde, Revuelta y Fernández, 2012), en el desarrollo y evaluación de competencias es relevante que un estudiante considere útil y valore lo que debe aprender. De esta forma existirán más posibilidades de que adquiera una práctica; en este caso que se apropie de las TIC para incorporarlas a contextos educativos. Por otra parte, al usar el portafolio los estudiantes ganarán experiencia en el manejo de herramientas digitales (Contreras-Higuera, Martinez-Olmo, Rubio-Hurtado y Vila-Banos, 2016).

A través de esta investigación se pudo establecer que los estudiantes de la carrera de Educación Física valoran el uso del portafolio en su formación, y esa valoración se traduce en aspectos tales como reconocer qué es útil y qué aprenden a través de él. De igual forma se pudieron identificar los factores que son considerados de valoración, tales como: que se genere interacción y colaboración; acceder a evidencias de otros compañeros; subir, organizar y respaldar evidencias. Así como también se logró identificar que el factor que afecta su uso es el diseño y aplicaciones del software.

Las mayores valoraciones son referidas a las posibilidades de interacción y colaboración que se generan entre ellos a partir de los espacios de comunicación (foro, blog, comentarios) y el acceso a material de evidencias tanto de productos, como de procesos que han sido puestos a disposición de la comunidad a través de los años. 
A partir de los hallazgos se evidenció que, a los estudiantes les desagrada el diseño y las características de algunas aplicaciones, principalmente lo referido a subir y organizar archivos.

Considerando lo obtenido en la investigación, los resultados serán compartidos con el Consejo de carrera para fortalecer el uso del portafolio electrónico, principalmente en aquellos módulos que son de la disciplina, y se replantearán algunos aspectos pedagógi- cos en relación a las demandas de aprendizaje que se hacen a través del portafolio, con la finalidad de reforzar procesos de reflexión y argumentación.

Por último, la percepción de utilidad y valoración expresada por los estudiantes, sobre una herramienta tecnológica, puede ser una experiencia favorable para su uso posterior como profesional.

\section{Referencias}

Agra, M., Gewerc, A. y Montero, L. (2003). El portafolios como herramienta de análisis en experiencias de formación on-line y presenciales. Enseñanza, 21, 19.

Aksit, F. (2016). Implementing Portfolios in Teacher Training: Why we use them and why we should usethem. Eurasian journal of educational research. 62. 97-u479

Armengol, J. et al. (2009). Experiencias sobre el uso del portafolio del estudiante en la UPC. RED, Revista de Educación a Distancia. Número monográfico VIII.- 30 de Abril de 2009. Número especial dedicado a Portafolios electrónicos y educación superior. Recuperado de: http:// www.um.es/ead/red/M8

Badia, A., Chumpitaz. L., Vargas, J. y Suárez, G. (2016). La percepción de la utilidad de la tecnología conforma su uso para enseñar y aprender. Revista Electrónica de Investigación Educativa, 18(3), 95-105. Recuperado de: http://redie.uabc.mx/redie/article/view/810

Barber, M., y Mourshed, M. (2008). Cómo hicieron los sistemas educativos con mejor desempeño del mundo para alcanzar sus objetivos. Recuperado de: http://www.mckinsey.com/ clientservice/socialsector/resources/pdf/Worlds_School_Systems_Final.pdf

Barbera, E. (2008). El Estilo e-portafolio. UOC. Barcelona.

Barret, H. (2012). Un modelo general para desarrollar e-portafolios con open source o herramientas web 2.0. Recuperado de: http://electronicportfolios.org/eportfolios/

Brun, M. (2011). Las tecnologías de la información y las comunicaciones en la formación inicial docente de América Latina. 72. Recuperado de: http://repositorio.cepal.org/bitstream/ handle/11362/6183/1/S1100626_es.pdf 
Chi-Cheng, Ch., Kuo-Hung, T, Chaoyun, L. y To-Yu Ch. (2013). Using e-portfolios to facilitate university students' knowledge management performance: E-portfolio vs. non-portfolio. Computers \& Educación. 69. 216-224. doi: 10.1016/j.compedu.2013.07.017

Chile, Ministerio de Educación. (2012). Estándares Orientadores para Carreras de Pedagogía en Educación Media. Recuperado de: http://www.cpeip.cl/usuarios/cpeip/File/librosestandaresvale/libromediafinal.pdf

Chile, Ministerio de Educación. (2012). ¿Qué es un Convenio de Desempeño? Recuperado de: http://www.mecesup.cl/index2.php?id_portal=59\&id_seccion=3605\&id_contenido=14986

Contreras-Higuera, W., Martinez-Olmo, F., Rubio-Hurtado, M. y Vila-Banos, R. (2016). University Students' perceptions of E-Portfolios and rubrics as comnedassessmenr tools in education courses. Journal of compunting in higher education. 54 (1). 85-107. doi: 10.1177/0735633115612784

Cotta, M., Minardi, R., da Costa, G. D. y de Mendonça, E. T. (2015). Portfólios crítico-reflexivos: uma proposta pedagógica centrada nascompetências cognitivas e metacognitivas. Interface-Comunicação, Saúde, Educação, 19(54).

Hepp, P. (2015). Desafíos de las políticas de integración de tecnologías en la formación inicial y continua de los docentes. En Mejorar los aprendizajes en la educación obligatoria: políticas y actores Instituto Internacional de planeamiento de la educación IIPE-UNESCO. Buenos Aires.

Hernández, R. Fernández, C., Baptista, P. (2010). Metodología de la investigación (5ªed.). Editorial Mc Graw Hill. México.

Kabilan, M. y Khan, M. (2012).Assessing pre-service English language teachers' learning using e-portfolios: Benefits, challenges and competencies gained. Computers \& education. 58 (4). 1007 - 1020. doi: 10.1016/j.compedu.2011.11.011

Koehler, M. y Mishra, P. (2009). What is technological pedagogical content knowledge? ContemporaryIssues in Technology and Teacher Education, 9(1), 60-70.

Perea, C. y Tarrés, M. (2012). Análisis del uso y apropiación social de un sistema de e-portafolios para la enseñanza y el aprendizaje. Revista del congrés internacional de docència universitària i innovació (cidui), 1(1).

Pérez, M. (2014). Evaluación de competencias mediante portafolios. Perspectiva Educacional, 53(1), 19-35. 
Oner, D. yAdadan,E. (2011). UseofWeb-Based Portfoliosas Toolsfor Reflection in Preservice Teacher Education. Journal of Teacher Education. 62 (5). 477-492. doi: 10.1177/0022487111416123

Oner, D. y Adadan, E. (2016). Are integred portfolio systems the answer? An evaluation of web-based portfolio system to improve preservice teachers' reflective thinking skills. Journal of compunting in higher education. 28(2). 236-260. doi: 10.1007/s12528-016-9108-y

Pozo, J. et al. (2006). Nuevas Formas de pensar la enseñanza y el aprendizaje. Las concepciones de profesores y alumnos. Grao. Barcelona.

Red Latinoamericana de Portales Educativos. (2013). Caracterización de Buenas Prácticas en la Formación Inicial Docente en TIC. Recuperado de: http://www.relpe.org/wpcontent/uploads/2013/04/12-Caracterizaci\%C3\%B3n-de-buenas-pr\%C3\%A1cticas-enformaci\%C3\%B3n-inicial-docente-en-TIC.pdf

Romero, C. (2008). El portafolio y el trabajo de grupo: una experiencia del crédito ECTS en la formación del maestro especialista en Educación Física. Revista electrónica interuniversitaria de formación del profesorado, 11(2), 6.

UNESCO. (1998). La Educación superior en el Siglo XXI. Visión y Acción. Recuperado de: http://www.unesco.org/education/educprog/wche/declaration_spa.htm

UNESCO. (2009). Conferencia Mundial sobre la Educación Superior -- 2009: La nueva dinámica de la educación superior y la investigación para el cambio social y el desarrollo. Recuperado de: http://www.unesco.org/education/WCHE2009/comunicado_es.pdf

UNESCO. (2013).Foro de alto nivel sobre políticas relativas a las TIC y la Educación para Todos: Logros alcanzados y perspectivas de avance. Recuperado de: http://www.unesco.org/new/ es/unesco/themes/icts/single-view/news/high_level_policy_forum_on_ict_and_education_ for_all_achievements_and_the_way_forward/\#.UsWfE_TuKVM

Valverde, J., Revuelta, F. y Fernández M. (2012). Modelos de evaluación por competencias a través de un sistema de gestión de aprendizaje. Experiencias en la formación inicial del profesorado. Revista Iberoamericana de Educación. No60 (51-62). Recuperado de: http://www.rieoei. org/rie60a03.pdf

Zeichner, K. (1995). Los Profesores como Profesionales Reflexivos y la Democratización de la Reforma Escolar. En Volver a Pensar la Educación: Prácticas y Discursos Educativos (Congreso Internacional de Didáctica). (pp 386-396). SegundaEdición, Volumen II. Morata. España. 